\title{
ON THE LYAPUNOV NUMBERS
}

\author{
SERGIǏ KOLYADA AND OLEKSANDR RYBAK
}

\begin{abstract}
We introduce and study the Lyapunov numbers - quantitative measures of the sensitivity of a dynamical system $(X, f)$ given by a compact metric space $X$ and a continuous map $f: X \rightarrow X$. In particular, we prove that for a minimal topologically weakly mixing system all Lyapunov numbers are the same.
\end{abstract}

\section{INTRODUCTION}

Throughout this paper $(X, f)$ denotes a topological dynamical system, where $X$ is a compact metric space with metric $d$ and $f: X \rightarrow X$ is a continuous map.

The notion of sensitivity (sensitive dependence on initial conditions) was first used by Ruelle [14. According to the works by Guckenheimer [10, Auslander and Yorke [5] a dynamical system $(X, f)$ is called sensitive if there exists a positive $\varepsilon$ such that for every $x \in X$ and every neighborhood $U_{x}$ of $x$, there exist $y \in U_{x}$ and a nonnegative integer $n$ with $d\left(f^{n}(x), f^{n}(y)\right)>\varepsilon$.

Recently several authors studied the different properties related to sensitivity (cf. Abraham et al.[1], Akin and Kolyada [3, Moothathu [13, Huang et al. [11]). The following proposition holds according to $[3]$.

Proposition 1.1. Let $(X, f)$ be a topological dynamical system. The following conditions are equivalent.

1. $(X, f)$ is sensitive.

2. There exists a positive $\varepsilon$ such that for every $x \in X$ and every neighborhood $U_{x}$ of $x$, there exists $y \in U_{x}$ with $\limsup _{n \rightarrow \infty} d\left(f^{n}(x), f^{n}(y)\right)>\varepsilon$.

3. There exists a positive $\varepsilon$ such that in any open $\Theta^{*} U$ in $X$ there are $x, y \in U$ and a nonnegative integer $n$ with $d\left(f^{n}(x), f^{n}(y)\right)>\varepsilon$.

4. There exists a positive $\varepsilon$ such that in any opene $U \subset X$ there are $x, y \in U$ with $\limsup _{n \rightarrow \infty} d\left(f^{n}(x), f^{n}(y)\right)>\varepsilon$.

For a dynamical system $(X, f)$ a point $x \in X$ is Lyapunov stable if the dependence of the orbit upon the initial position is continuous at $x$ (see [3]). This is most easily defined using the $f$-extension of the metric $d$ :

$$
d_{f}(x, y)=\sup \left\{d\left(f^{n}(x), f^{n}(y)\right): n \geq 0\right\}
$$

for $x, y \in X$. Clearly, $d_{f}$ is a metric on $X$ and

$$
d_{f}(x, y)=\max \left[d(x, y), d_{f}(f(x), f(y))\right] .
$$

Using these metrics we define the diameter and $f$-diameter for $A \subset X$, the radius and $f$-radius for a neighborhood $U_{x}$ of a point $x \in X$

$$
\begin{array}{r}
\operatorname{diam}(A)=\sup \{d(x, y): x, y \in A\}, \operatorname{diam}_{f}(A)=\sup \left\{d_{f}(x, y): x, y \in A\right\}, \\
\operatorname{rad}\left(U_{x}\right)=\sup \left\{d(x, y): y \in U_{x}\right\}, \operatorname{rad}_{f}\left(U_{x}\right)=\sup \left\{d_{f}(x, y): y \in U_{x}\right\} .
\end{array}
$$

2010 Mathematics Subject Classification. Primary 37B05; Secondary 54H20, 37B25.

Key words and phrases. Lyapunov numbers, minimal map, sensitive dynamical system, topologically weakly mixing map.

${ }^{*}$ Because we so often have to refer to open, nonempty subsets, we will call such subsets opene. 
The topology obtained from the metric $d_{f}$ is usually strictly coarser than the original $d$ topology. When we use a term like "open", we refer exclusively to the original topology.

A point $x \in X$ is called Lyapunov stable if for every $\varepsilon>0$ there exists a $\delta>0$ such that $\operatorname{rad}\left(U_{x}\right)<\delta$ implies $\operatorname{rad}_{f}\left(U_{x}\right) \leq \varepsilon$. This condition says exactly that the sequence of iterates $\left\{f^{n}: n \geq 0\right\}$ is equicontinuous at $x$. Hence, such a point is also called an equicontinuity point. We label associated point sets:

$$
\begin{array}{r}
\operatorname{Eq}_{\varepsilon}(f)=\bigcup\left\{U_{x} \subset X: U_{x} \text { is a neighborhood of a point } x\right. \\
\text { with } \left.\operatorname{rad}_{f}\left(U_{x}\right) \leq \varepsilon\right\} \text { and } \operatorname{Eq}(f)=\bigcap_{\varepsilon>0} \operatorname{Eq}_{\varepsilon}(f) .
\end{array}
$$

As the label suggests, $\operatorname{Eq}(f)$ is the set of equicontinuity points. If $\operatorname{Eq}(f)=X$, i.e. every point is equicontinuous, then the two metrics $d$ and $d_{f}$ are topologically equivalent and so, by compactness, they are uniformly equivalent. Such a system is called equicontinuous. Thus, $(X, f)$ is equicontinuous exactly when the sequence $\left\{f^{n}: n \geq 0\right\}$ is uniformly equicontinuous.

If the $G_{\delta}$ set $\operatorname{Eq}(f)$ is dense in $X$ then the system is called almost equicontinuous. On the other hand, if $\operatorname{Eq}_{\varepsilon}(f)=\varnothing$ for some $\varepsilon>0$ then it is the same that the system shows sensitive dependence upon initial conditions or, more simply, $(X, f)$ is sensitive. We define

$\mathbb{L}_{r}:=\sup \left\{\varepsilon:\right.$ for every $x \in X$ and every neighborhood $U_{x}$ of $x$ there exist $y \in U_{x}$ and a nonnegative integer $n$ with $\left.d\left(f^{n}(x), f^{n}(y)\right)>\varepsilon\right\}$

and call it the (first) Lyapunov number.

It can happen that $\operatorname{Eq}_{\varepsilon}(f) \neq \varnothing$ for all positive $\varepsilon$ and yet still the intersection, $\mathrm{Eq}(f)$, is empty (see [3]). This cannot happen when the system is transitive (Glasner and Weiss [9], Akin et al [2]).

Theorem 1.2. Let $(X, f)$ be a topologically transitive dynamical system. Exactly one of the following two cases holds.

Case $\mathbf{i}(\operatorname{Eq}(f) \neq \varnothing)$ Assume there exists an equicontinuity point for the system. The equicontinuity points are exactly the transitive points, i.e. $\mathrm{Eq}(f)=\operatorname{Trans}(f)$, and the system is almost equicontinuous. The map $f$ is a homeomorphism and the inverse system $\left(X, f^{-1}\right)$ is almost equicontinuous. Furthermore, the system is uniformly rigid meaning that some subsequence of $\left\{f^{n}: n=0,1, \ldots\right\}$ converges uniformly to the identity.

Case $\mathrm{ii}(\mathrm{Eq}(f)=\varnothing)$ Assume the system has no equicontinuity points. The system is sensitive, i.e. there exists $\varepsilon>0$ such that $\operatorname{Eq}_{\varepsilon}(f)=\varnothing$.

Corollary 1.3. If $(X, f)$ is a minimal dynamical system then it is either sensitive or equicontinuous.

Let us define

$$
\operatorname{Equ}_{\varepsilon}(f)=\bigcup\left\{U \subset X: U \text { is open with } \operatorname{diam}_{f}(U) \leq \varepsilon\right\} .
$$

Obviously $\operatorname{Eq}(f)=\cap_{\varepsilon>0} \operatorname{Equ}_{\varepsilon}(f)$, and if $\operatorname{Equ}_{\varepsilon}(f)=\varnothing$ for some $\varepsilon>0$ then the system $(X, f)$ is sensitive (see also Proposition 1.1). Therefore, it is natural to define

$$
\mathbb{L}_{d}:=\sup \{\varepsilon: \text { in any opene } U \subset X \text { there exist } x, y \in U \text { and there is }
$$

a positive integer $n$ with $\left.d\left(f^{n}(x), f^{n}(y)\right)>\varepsilon\right\}$

and call it the second Lyapunov number.

${ }^{\dagger}$ We recall the definition in Section 3. 
According to Proposition 1.1 we will define

$$
\begin{array}{r}
\overline{\mathbb{L}}_{r}:=\sup \left\{\varepsilon: \text { for every } x \in X \text { and every open neighborhood } U_{x} \text { of } x\right. \\
\text { there exists } \left.y \in U_{x} \text { with } \limsup _{n \rightarrow \infty} d\left(f^{n}(x), f^{n}(y)\right)>\varepsilon\right\}, \\
\overline{\mathbb{L}}_{d}:=\sup \{\varepsilon: \text { in any opene } U \subset X \text { there exist } x, y \in U \text { with } \\
\left.\limsup _{n \rightarrow \infty} d\left(f^{n}(x), f^{n}(y)\right)>\varepsilon\right\} .
\end{array}
$$

Sometimes it will be useful to use also the following notations

$$
\mathbb{L}_{1}:=\mathbb{L}_{r} ; \mathbb{L}_{2}:=\mathbb{L}_{d} ; \mathbb{L}_{3}:=\overline{\mathbb{L}}_{r} ; \mathbb{L}_{4}:=\overline{\mathbb{L}}_{d}
$$

So, various definitions of sensitivity, formally give us different Lyapunov numbers - quantitative measures of these sensitivities.

In Section 2 we prove that for a topological dynamical system $(X, f)$, it holds $\mathbb{L}_{d} \leq 2 \overline{\mathbb{L}}_{r}$. In Section 3 we examine the equalities between the Lyapunov numbers for topologically transitive systems and in Section 4 for weakly mixing systems. In particular, we prove that for topologically weakly mixing minimal systems all Lyapunov numbers are the same. Finally, in Section 5 we give some examples and open problems for Lyapunov numbers.

Acknowledgements. We thank the anonymous reviewer for helpful remarks and suggestions. The first author was supported by Max-Planck-Institut für Mathematik (Bonn); he acknowledges the hospitality of the Institute.

\section{A general inequality FOR the Lyapunov nUmbers}

Directly from the definitions, the following inequalities hold

$$
\mathbb{L}_{d} \geq \overline{\mathbb{L}}_{d} \geq \overline{\mathbb{L}}_{r} \text { and } \mathbb{L}_{d} \geq \mathbb{L}_{r} \geq \overline{\mathbb{L}}_{r} .
$$

Proposition 2.1. Let $(X, f)$ be a sensitive topological dynamical system. Then $\mathbb{L}_{d} \leq 2 \overline{\mathbb{L}}_{r}$.

Proof. Let $\mathbb{L}_{d}$ be the second Lyapunov number of $(X, f)$. Fix a (small enough) $\delta>0$, a point $x \in X$ and a neighborhood $U_{x}$ of $x$. Let $U_{0}=U_{x}$ and $n_{0}$ be the first positive integer, for which $\operatorname{diam}\left(f^{n_{0}}\left(U_{0}\right)\right)>\mathbb{L}_{d}-\delta$. There exists a point $y_{0} \in U_{0}$ such that $d\left(f^{n_{0}}(x), f^{n_{0}}\left(y_{0}\right)\right)>\left(\mathbb{L}_{d}-\delta\right) / 2$. Choose an opene $U_{1}$ with its closure contained in $U_{0}$ such that $y_{0} \in U_{1}$ and $\operatorname{diam}\left(f^{m}\left(U_{1}\right)\right) \leq \delta / 2$ for every non-negative integer $m \leq n_{0}$. Let $n_{1}$ be the first positive integer, for which $\operatorname{diam}\left(f^{n_{1}}\left(U_{1}\right)\right)>$ $\mathbb{L}_{d}-\delta$. By the definition of $U_{1}$, we clearly have $n_{1}>n_{0}$.

We define recursively opene sets $U_{2}, U_{3}, \ldots$ and positive integers $n_{2}, n_{3}, \ldots$ as follows. Since $n_{k-1}$ is defined, there exists a point $y_{k-1} \in U_{k-1}$ such that $d\left(f^{n_{k-1}}(x), f^{n_{k-1}}\left(y_{k-1}\right)\right)>\left(\mathbb{L}_{d}-\delta\right) / 2$. So we can choose an opene $U_{k}$ in $U_{k-1}$ such that $y_{k-1} \in U_{k}$ and $\operatorname{diam}\left(f^{m}\left(U_{n_{k}}\right)\right) \leq \delta / 2$ for every non-negative integer $m \leq n_{k-1}$. Let $n_{k}$ be the first positive integer, for which $\operatorname{diam}\left(f^{n_{k}}\left(U_{k}\right)\right)>\mathbb{L}_{d}-\delta$. As in the previous step, by the definition of $U_{k}$ we clearly have $n_{k}>n_{k-1}$.

If $y$ is a point of the nonempty intersection $\cap_{k} \overline{U_{n_{k}}}$, then, obviously, $y \in U$ and $\lim \sup _{n \rightarrow \infty} d\left(f^{n}(x), f^{n}(y)\right) \geq \mathbb{L}_{d} / 2-\delta$.

As a consequence of the inequalities at the beginning of Section 2 and Proposition 2.1 we conclude that $\mathbb{L}_{i} \leq 2 \mathbb{L}_{j}$ for any $i, j \in\{1,2,3,4\}$.

\section{LYAPUNOV NUMBERS FOR TRANSITIVE MAPS}

A topological dynamical system $(X, f)$ is called topologically transitive, if for any pair of opene subsets $U, V \in X$

$$
n_{f}(U, V):=\left\{n \in \mathbb{Z}_{+}: U \cap f^{-n}(V) \neq \varnothing\right\}
$$


is infinite. A point $x \in X$ is called a transitive point if its orbit $\left\{x, f(x), f^{2}(x), \ldots\right\}$ is dense in $X$. If $(X, f)$ is topologically transitive and $X$ is compact, then the set of transitive points is a $G_{\delta}$-dense subset of $X$.

If every point of a dynamical system $(X, f)$ is transitive, then this system is called minimal. An $f$-invariant closed subset $M \subset X$ is called minimal if the orbit of any point of $M$ is dense in $M$ (in this case a point of $M$ is called minimal, too).

For a dynamical system $(X, f)$, a point $x \in X$ and a set $U \subset X$ let

$$
n_{f}(x, U):=\left\{n \in \mathbb{Z}_{+}: f^{n}(x) \in U\right\} .
$$

A point $x \in X$ is said to be recurrent if for every neighborhood $U$ of $x$ the set $n_{f}(x, U)$ is infinite.

A subset $S$ of $\mathbb{Z}_{+}$is syndetic if it has bounded gaps, i.e. there is $N \in \mathbb{N}$ such that $\{i, i+1, \ldots, i+N\} \cap S \neq \varnothing$ for every $i \in \mathbb{Z}_{+} . S$ is thick if it contains arbitrarily long runs of positive integers, i.e. there is a strictly increasing subsequence $\left\{n_{i}\right\}$ such that $S \supset \cup_{i=1}^{\infty}\left\{n_{i}, n_{i}+1, \ldots, n_{i}+i\right\}$.

Some dynamical properties can be interpreted by using the notions of syndetic or thick subsets. For example, a classic result of Gottschalk states that $x \in X$ is a minimal point if and only if $n_{f}(x, U)$ is syndetic for any neighborhood $U$ of $x$, and a topological dynamical system $(X, T)$ is (topologically) weakly mixing (we recall the definition in Section 4$)$ if and only if $n_{f}(U, V)$ is thick for any opene subsets $U, V$ of $X$ [7, 8 .

Theorem 3.1. Let $(X, f)$ be a sensitive topologically transitive dynamical system. Then $\mathbb{L}_{d}=\overline{\mathbb{L}}_{d}$.

Proof. By the definition of $\mathbb{L}_{d}$, for any $\varepsilon<\mathbb{L}_{d}$ and for any opene $U \in X$ there are points $x, y \in U$ and a positive integer $n_{0}$ such that $d\left(f^{n_{0}}(x), f^{n_{0}}(y)\right)>\varepsilon$. Choose an arbitrary (small) $\delta>0$. Let $U_{x}, U_{y} \subset U$ be neighborhoods of $x$ and $y$ such that $\operatorname{diam}\left(f^{n_{0}}\left(U_{x}\right)\right)<\delta$ and $\operatorname{diam}\left(f^{n_{0}}\left(U_{y}\right)\right)<\delta$. If $z \in U_{x}$ is a transitive point, there is a positive integer $m$ for which $f^{m}(z) \in U_{y}$. By the triangle inequality we have $d\left(f^{n_{0}}(z), f^{n_{0}+m}(z)\right)>\varepsilon-2 \delta$.

Let $U_{z}$ be a neighborhood of $z$ such that $U_{z} \subset U_{x}$ and $f^{m}\left(U_{z}\right) \subset U_{y}$. Then obviously $\operatorname{diam}\left(f^{n_{0}}\left(U_{z}\right)\right)<\delta$ and $\operatorname{diam}\left(f^{n_{0}+m}\left(U_{z}\right)\right)<\delta$. Since a sensitive system has no isolated points, $U_{z}$ is infinite. Therefore, the orbit of the point $z$ visits $U_{z}$ infinitely many times. If $n_{k}$ is such that $f^{n_{k}}(z) \in U_{z}$, then $f^{n_{0}+n_{k}}(z)=f^{n_{0}}\left(f^{n_{k}}(z)\right) \subset$ $f^{n_{0}}\left(U_{z}\right)$ and $f^{n_{0}+n_{k}+m}(z)=f^{n_{0}+m}\left(f^{n_{k}}(z)\right) \subset f^{n_{0}+m}\left(U_{z}\right)=f^{n_{0}}\left(f^{m}\left(U_{z}\right)\right) \subset$ $f^{n_{0}}\left(U_{y}\right)$. And so, by the triangle inequality, $d\left(f^{n_{0}+n_{k}}(z), f^{n_{0}+n_{k}+m}(z)\right)>\varepsilon-2 \delta$. From this we have $\overline{\mathbb{L}}_{d}>\lim \sup _{n \rightarrow \infty} d\left(f^{n}(z), f^{n}\left(f^{m}(z)\right)\right) \geq \varepsilon-2 \delta$. Since $\delta>0$ and $\varepsilon<\mathbb{L}_{d}$ were chosen arbitrarily, $\mathbb{L}_{d}=\overline{\mathbb{L}}_{d}$.

A topologically transitive dynamical system $(X, f)$, where $X$ has no isolated points, is called ToM if every point $x \in X$ is either (topologically) transitive or minimal. ToM systems were introduced by Downarowicz and Ye in 6 . Since we do not require that both types are present (as in [6]), a minimal system is also ToM. If a ToM system is not minimal, then the set of minimal points is dense in $X$ (because for a transitive, but non-minimal system, the set of non-transitive points is dense (see for instance [12])).

Theorem 3.2. Let $(X, f)$ be a sensitive ToM system. Then $\mathbb{L}_{r}=\overline{\mathbb{L}}_{r}$.

Proof. Fix a point $x \in X$. Let $U_{x}$ be a neighborhood of $x$ and let $\delta>0$. By the definition of $\mathbb{L}_{r}$, there exist a point $y \in U_{x}$ and a positive integer $m$ such that $d\left(f^{m}(x), f^{m}(y)\right)>\mathbb{L}_{r}-\delta$. Take a neighborhood $U_{y} \subset U_{x}$ of point $y$ such that $\operatorname{diam} f^{m}\left(U_{y}\right)<\delta$. 
Now, if $x$ is a transitive point, one can just repeat the idea of the proof of Theorem 3.1 for the proof of this case. If $x$ is not transitive, then is minimal. Since $(X, f)$ is ToM, we can find a minimal point $z_{1} \in U_{y}$ and therefore $d\left(f^{m}(x), f^{m}\left(z_{1}\right)\right)>\mathbb{L}_{r}-2 \delta$.

Consider the direct product system $\left(\overline{\mathrm{Orb}_{f}(x)} \times \overline{\mathrm{Orb}_{f}\left(z_{1}\right)},\left.f\right|_{\overline{\mathrm{Orb}_{f}(x)}} \times\left. f\right|_{\overline{\mathrm{Orb}_{f}\left(z_{1}\right)}}\right)$. Let $M$ be a minimal subset of this system. Then obviously $M \cap M_{x} \neq \varnothing$, where $M_{x}:=\left\{(x, z): z \in \overline{\operatorname{Orb}_{f}\left(z_{1}\right)}\right\}$. Hence there is a point $\left(x, z_{2}\right) \in U_{x} \times \overline{\operatorname{Orb}_{f}\left(z_{1}\right)}$, which is minimal, and therefore (uniformly) recurrent for the map $\left.f\left|\overline{\operatorname{Orb}_{f}(x)} \times f\right|_{\operatorname{Orb}_{f}\left(z_{1}\right)}\right)$. Clearly, every point of the form $\left(x, f^{k}\left(z_{2}\right)\right), k=0,1,2, \ldots$ will be uniformly recurrent too. Since $z_{1}$ is minimal, we can take a positive integer $k$, such that $z_{3}:=f^{k}\left(z_{2}\right) \in$ $U_{y}$. Therefore, we have $\limsup _{n \rightarrow \infty} d\left(f^{n}(x), f^{n}\left(z_{3}\right)\right) \geq \mathbb{L}_{r}-2 \delta$. Since $x$ and $\delta>0$ were chosen arbitrarily, we get $\mathbb{L}_{r}=\overline{\mathbb{L}}_{r}$.

As a corollary of the last two theorems we conclude that the equalities $\mathbb{L}_{r}=\overline{\mathbb{L}}_{r}$ and $\mathbb{L}_{d}=\overline{\mathbb{L}}_{d}$ hold for minimal dynamical systems. And what we can say about dynamical systems for which $\mathbb{L}_{r}=\mathbb{L}_{d}$ holds?

\section{LYAPUNOV NUMBERS FOR WEAKLY MIXING MAPS}

Recall that a topological dynamical system $(X, f)$ is called (topologically) weakly mixing if for any opene $U_{1}, U_{2}, V_{1}, V_{2} \in X$ there is a non-negative integer $n$ such that $U_{1} \cap f^{-n}\left(V_{1}\right) \neq \varnothing$ and $U_{2} \cap f^{-n}\left(V_{2}\right) \neq \varnothing$. In other words if its direct product $(X \times X, f \times f)$ is topologically transitive.

Theorem 4.1. Let $(X, f)$ be a topologically weakly mixing dynamical system. Then

1. $\mathbb{L}_{d}=\overline{\mathbb{L}}_{d}=\operatorname{diam}(X)$.

2. $\mathbb{L}_{r}=\overline{\mathbb{L}}_{r}$.

3. If, in addition, $(X, f)$ is minimal, then $\mathbb{L}_{r}=\overline{\mathbb{L}}_{r}=\mathbb{L}_{d}=\overline{\mathbb{L}}_{d}=\operatorname{diam}(X)$.

Proof. 1. Since a weakly mixing system is topologically transitive, from Theorem 3.1 we have $\mathbb{L}_{d}=\overline{\mathbb{L}}_{d}$. Since $(X, f)$ be a topologically weakly mixing, also the direct product $(X \times X, f \times f)$ is topologically transitive. So, in every open set in the product, in particular, in the Cartesian square of every ball $U$ in $X$, there is a transitive point of $(X \times X, f \times f)$, i.e., a pair of points $x, y \in U$. Such pair visits all places in the Cartesian square $X \times X$ infinitely many times. It means that $\limsup \sup _{n \rightarrow \infty} d\left(f^{n}(x), f^{n}(y)\right)=\operatorname{diam}(X)=\overline{\mathbb{L}}_{d}$.

2. Let $x \in X$. Since $(X, f)$ is weakly mixing, there is a point $z \in X$, such that for any neighborhood $G$ of $z$ and any opene $U, V$ in $X$ there exist infinitely many positive integers $n$, for which $f^{n}(x) \in G$ and $f^{n}(U) \cap V \neq \varnothing([3])$.

By the definition of $\mathbb{L}_{r}$, for the point $z$ and any (small enough) positive $\delta$ there is a point $y \in X$ and a positive integer $k$ such that $d\left(f^{k}(y), f^{k}(z)\right)>\mathbb{L}_{r}-\delta$.

Now, let $U_{x}$ be a neighborhood of point $x$, let $G_{z}$ and $V_{y}$ be open balls of radius $\delta$ centered at points $f^{k}(z)$ and $f^{k}(y)$, respectively. Suppose also $G_{z} \cap V_{y}=\varnothing$. In order to prove the second part of this theorem we will find a point in $U_{x}$ by using the above property from [3]. Let $n_{0}$ be a positive integer such that $f^{n_{0}}(x) \in G_{z}$ and $f^{n_{0}}\left(U_{x}\right) \cap V_{y} \neq \varnothing$. Put $U_{0}:=U_{x} \cap f^{-n_{0}}\left(V_{y}\right)$. Obviously, $U_{0}$ is an opene subset of $U_{x}$, $\overline{U_{0}} \subset \overline{U_{x}}$ and $x \notin U_{0}$. Define inductively opene sets $U_{1}, U_{2}, \ldots$ and positive integers $n_{i}$ as follows. Let $n_{k}$ be a (large enough) positive integer, say $n_{k} \geq k$, such that $f^{n_{k}}(x) \in G_{z}$ and $f^{n_{k}}\left(U_{k-1}\right) \cap V_{y} \neq \varnothing$. Define $U_{k}:=U_{k-1} \cap f^{-n_{k}}\left(V_{y}\right)$. It is clear that $U_{k}$ is an opene subset in $X$ and $U_{i} \subset U_{i-1}$ for any $i \geq 1$. Hence $\overline{U_{0}} \supset \overline{U_{1}} \supset \overline{U_{2}} \supset \ldots$ If $u$ is a point of the nonempty intersection $\cap_{i} \overline{U_{i}}$, then for any natural $i$ we have $f^{n_{i}}(u) \in \bar{V}_{y}$ and $f^{n_{i}}(x) \in G_{z}$. Therefore, $\limsup _{n \rightarrow \infty} d\left(f^{n}(x), f^{n}(u)\right) \geq \mathbb{L}_{r}-3 \delta$. Since $\delta>0$ is arbitrary, we have $\mathbb{L}_{r}=\overline{\mathbb{L}}_{r}$.

3. Cases 1 and 2 imply that it is sufficient to prove $\mathbb{L}_{r}=\operatorname{diam}(X)$. Let $x \in X$ and let $U_{x}$ be a neighborhood of $x$. There are two opene (infinite) sets $V_{x}$ and $V_{y}$ 
in $X$ and a positive (small enough) number $\delta$ such that the distance between $V_{x}$ and $V_{y}$ is large or equal to $\operatorname{diam}(X)-\delta$.

As we have mentioned before, since $\operatorname{TDS}(X, f)$ is minimal, any point of $X$ is uniformly recurrent. In particular, it means that $n_{f}\left(x, V_{x}\right)$ is a syndetic subset of $\mathbb{Z}_{+}$. On the other hand $(X, f)$ is also a topologically weakly mixing dynamical system. And again it means that $n_{f}\left(U, V_{y}\right)$ is a thick subset of $\mathbb{Z}_{+}$. Hence $n_{f}\left(x, V_{x}\right) \cap n_{f}\left(U, V_{y}\right) \neq \varnothing$ and therefore there exist a point $y \in U$ and a positive integer $k \in n_{f}\left(x, V_{x}\right) \cap n_{f}\left(U, V_{y}\right)$ such that $f^{k}(x) \in V_{x}$ and $f^{k}(y) \in V_{y}$. So, $d\left(f^{k}(x), f^{k}(y)\right) \geq \operatorname{diam}(X)-\delta$. Since $\delta>0$ was arbitrary, we get $\mathbb{L}_{r}=$ $\operatorname{diam}(X)$.

\section{Concluding Remarks}

Firstly, let us remark that there are topologically weakly mixing (even topologically mixing) systems for which $\mathbb{L}_{r}=\overline{\mathbb{L}}_{r}=\operatorname{diam}(X) / 2$. For instance, the continuous interval map $g:[0,1] \rightarrow[0,1]$, where $g(x)=3((x-1 / 3)-|x-1 / 3|+|x-2 / 3|)$, is topologically mixing, one of its fixed points is $1 / 2$, therefore clearly that $\mathbb{L}_{r}=1 / 2$.

Also (as we will see in Proposition 5.1 below) there are dynamical systems for which $\mathbb{L}_{r}=2 \overline{\mathbb{L}}_{r}$, but it is still an open question for topologically transitive maps (non-minimal by Theorem 3.2).

Two more open questions:

1. Does there exist a non-transitive dynamical system $(X, f)$ for which $\mathbb{L}_{d}>$ $\overline{\mathbb{L}}_{d}$ and/or $\mathbb{L}_{r}>\overline{\mathbb{L}}_{d}$ ?

2. Does there exist a minimal dynamical system $(X, f)$ for which $\mathbb{L}_{d}>\mathbb{L}_{r}$ ?

Proposition 5.1. There exists a topological dynamical system $(X, f)$ for which $\mathbb{L}_{r}=2 \overline{\mathbb{L}}_{r}$.

Proof. We define the space $X$ as a compact surface in $\mathbb{R}^{3}$ which is homeomorphic to a two-dimensional disk in $\mathbb{R}^{2}$. More precisely, the cylindric coordinates of a point $(x, y, z) \in X$ have the form $(r, \varphi, z)$, where $r=\sqrt{x^{2}+y^{2}}$ and $\varphi$ is an angle, for which $x=r \cos \varphi$ and $y=r \sin \varphi$. In other words, $(r, \varphi)$ are the polar coordinates of $(x, y)$, and $z$ remains unchanged. Let $h(r)=8 r(1-r)$. Now, define $X$ as a set of points with cylindric coordinates $(r, \varphi, h(r))$, where $0 \leq r \leq 1, \varphi \in \mathbb{R}$, and let the Euclidian metric (in $\mathbb{R}^{3}$ ) $d$ be the metric on $X$.

Now we define a continuous map $f$ from $X$ to itself as follows $f:(r, \varphi, h(r)) \rightarrow$ $(g(r), 2 \varphi, h(g(r)))$, where $g(x)$ is a continuous map $[0,1] \rightarrow[0,1]$ with $g(0)=0$, $g(1)=1$ and $g(x)>x$ for all $x \in(0,1)$. From this properties one can easily deduce that $\lim _{n \rightarrow \infty} g^{n}(x)=1$ for any $x \in(0,1]$. For example, let $g(x)=2 x-x^{2}$.

Let $p \in X$ and $U$ be a neighborhood of $p$. If $p \neq(0,0,0)$, then for any $\delta>0$ there are $n \in \mathbb{N}$ and $q \in U$ such that $d\left(f^{n}(p), f^{n}(q)\right)>2-\delta$. If $p=(0,0,0)$, then there are $n \in \mathbb{N}$ and $q \in U$, for which $f^{n}(q)$ lies on a circumference of $X$ with the center $(0,0,2)$ (in $\left.\mathbb{R}^{3}\right)$ and the radius $\frac{1}{2}$. For these $n$ and $q$ we have $d\left(f^{n}(p), f^{n}(q)\right)>2$ and so $\mathbb{L}_{r} \geq 2$.

Now, let $p=(0,0,0)$. The equality $\lim _{n \rightarrow \infty} d\left(f^{n}(p), f^{n}(q)\right)=1$ holds for any $q \neq p$. So $\overline{\mathbb{L}}_{r} \leq 1$. Since $\mathbb{L}_{r} \leq 2 \overline{\mathbb{L}}_{r}$ (by Proposition 2.1 ), it gives $\mathbb{L}_{r}=2 \overline{\mathbb{L}}_{r}$.

The idea of introducing and studying the Lyapunov numbers is derived from the following:

1. If some practical assumption holds for the behavior of a particular system, for example, a physical object, we need to know how far we can go wrong in calculations, if we mean to predict the evolution of the system over a quite long term. Only knowing that there could exist errors in the calculations of the future behavior of a system is not that useful, since from the practical point of view, 
the existence of errors in calculations of almost all natural systems (as a result of inaccurate initial data) is a well-known fact. So, quantitative analysis of sensitivity that determines to what extent one's calculations are accurate is of great interest. Comparison of different Lyapunov numbers (the ones which are determined by the upper limit and the ones without limit) demonstrates that errors in calculations cannot disappear (decrease) during passing of time. That is, we cannot expect that, for example, after 10000 or 1000000 steps the accuracy of our prediction increase significantly (which seems commonsensical).

2. According to the Auslander theorem, one of the most important theorems in topological dynamics, any proximal cell (i.e., $\operatorname{Prox}_{f}(x):=\{y \in X$ : $\left.\left.\liminf _{n \rightarrow \infty} d\left(f^{n}(x), f^{n}(y)\right)=0\right\}\right)$ contains a minimal point [4. This implies, in particular, that a distal point is always minimal. It should be noted that, if $(X, f)$ is a weak mixing dynamical system then for every $x \in X$, the proximal cell $\operatorname{Prox}_{f}(x)$ is dense in $X$ [3]. What about this property for the sensitive topologically transitive systems, in particular, for the Devaney systems (i.e., topologically transitive with a dense set of periodic points systems)? There is a direct connection between this question and the following one: When does $\mathbb{L}_{r}=\overline{\mathbb{L}}_{r}$ hold for a sensitive topologically transitive system?

\section{REFERENCES}

[1] Ch. Abraham, G. Biau and B. Cadre, On Lyapunov exponent and sensitivity, J. Math. Anal. Appl. 290 (2004), 395-404.

[2] E. Akin, J. Auslander and K. Berg, When is a transitive map chaotic?, Convergence in ergodic theory and probability (Columbus, OH, 1993), 25-40, Ohio State Univ. Math. Res. Inst. Publ., 5(1996), deGruyter, Berlin.

[3] E. Akin and S. Kolyada, Li-Yorke sensitivity, Nonlinearity 16 (2003), 1421-1433.

[4] J. Auslander, Minimal flows and their extensions, North-Holland Mathematics Studies, 153. North-Holland Publishing Co., Amsterdam, 1988.

[5] J. Auslander and J. Yorke, Interval maps, factors of maps and chaos, Tohoku Math. J. 32 (1980), 177-188.

[6] T. Downarowicz and X. Ye, When every point is either transitive or periodic, Colloq. Math. 93(2002), 137-150.

[7] H. Furstenberg, Disjointness in ergodic theory, minimal sets, and a problem in Diophantine approximation, Math. Systems Theory, 1(1967), 1-49.

[8] H. Furstenberg, Recurrence in ergodic theory and combinatorial number theory, M. B. Porter Lectures. Princeton University Press, Princeton, N.J., 1981.

[9] E. Glasner and B. Weiss, Sensitive dependence on initial conditions, Nonlinearity, 6(1993), $1067-1075$.

[10] J. Guckenheimer, Sensitive dependence to initial conditions for one-dimensional maps. Comm. Math. Phys. 70(1979), 133-160.

[11] W. Huang, P. Lu and X. Ye, Measure-theoretical sensitivity and equicontinuity. Israel J. Math. 183(2011), 233-283.

[12] S. Kolyada and L. Snoha, Some aspects of topological transitivity - a survey, Iteration theory (ECIT 94, Opava), 3-35, Grazer Math. Ber., 334, Karl-Franzens-Univ. Graz, Graz, 1997.

[13] T.K.S. Moothathu, Stronger forms of sensitivity for dynamical systems, Nonlinearity 20 (2007), 2115-2126.

[14] D. Ruelle, Dynamical systems with turbulent behavior. Mathematical problems in theoretical physics (Proc. Internat. Conf., Univ. Rome, Rome, 1977), 341-360, Lecture Notes in Phys., 80, Springer, Berlin-New York, 1978.

Institute of Mathematics, NASU, Tereshchenkivs'Ka 3, 01601 Kyiv, Ukraine

E-mail address: skolyada@imath.kiev.ua

Institute of Mathematics, NASU, Tereshchenkivs'Ka 3, 01601 Kyiv, Ukraine

E-mail address: semperfi@ukr.net 